J. Gynäkol. Endokrinol. AT 2019 • 29:13-22 https://doi.org/10.1007/s41974-019-0085-9 Online publiziert: 19. März 2019

(c) Der/die Autor(en) 2019

\author{
Linda Mayr ${ }^{1} \cdot$ Dimitar Georgiev $^{2} \cdot$ Albena Toulev $^{3}$ \\ 'Wien, Österreich \\ ${ }^{2}$ Institute for Medical Research, Sofia, Bulgarien \\ ${ }^{3}$ Zürich, Schweiz
}

\title{
Eine Proof-of-concept-Studie von Isoflavandiol-E55-RS-Vaginal- kapseln oder Vaginalgel zur Linderung der menopausalen Vaginalatrophie
}

\section{Einleitung}

Die Menopause ist durch einen verringerten Östrogenspiegel im weiblichen Körper gekennzeichnet. Aufgrund der verminderten Östrogenbildung im vulvovaginalen Gewebe können verschiedene vaginale Symptome wie Dyspareunie, vaginale Trockenheit, vaginaler Juckreiz und Libidoverlust auftreten [1, 2]. Diese Symptome können im Begriff Urogenitalsyndrom der Menopause, ehemalige vulvovaginale Atrophie (VVA; [1, 3]), zusammengefasst werden. Infolge des Östrogenmangels erhöht sich der vaginale $\mathrm{pH}$ Wert auf 6-7, und es kann eine Veränderung der vaginalen Lactobacillus-Kolonisation beobachtet werden [4]. Darüber hinaus kommt es, bedingt durch den verminderten Östrogenspiegel, zu einer Verdünnung des Vaginalepithels. Zusätzlich kann eine Verschiebung der vaginalen Epithelzellen beobachtet werden. So nimmt bei menopausalen Frauen die Anzahl an superfizialen Zellen ab und parabasale Zellen nehmen zu [5]. Laut der International Menopause Society leiden bis zu $40 \%$ der postmenopausalen Frauen an vaginalen Beschwerden. Obwohl es verschiedene wirksame Behandlungsmethoden gibt, suchen nur $25 \%$ der Betroffenen nach Hilfe [6]. Vaginale Atrophie ist eine Erkrankung, die nicht nur bei postmenopausalen Frauen auftritt, aber die Prävalenz ist in dieser Gruppe am höchsten und tritt in den meisten Fäl- len 4-5 Jahre nach der Menopause auf [6]. Um den Grad von VVA als einen numerischen Wert auszudrücken, ist es möglich, den Reifungswert (MV; [7]) zu berechnen.

Da die lokale Behandlung mit hormonhaltigen Präparaten umstritten ist und viele Frauen keine Hormontherapie zur Behandlung dieser vaginalen Symptome anwenden wollen, sind andere Therapieformen erforderlich. Eine mögliche und gut akzeptierte Methode wäre die vaginale Behandlung mit Equol [8]. Equol gehört zur Familie der Isoflavone, einer Untergruppe der Phytoöstrogene, welche sekundäre Verbindungen in verschiedenen Pflanzen, wie zum Beispiel im grünen Tee oder Sojabohnen, sind. Die strukturelle Ähnlichkeit zu $17 \beta$-Östradiol ermöglicht die Interaktion dieser Verbindungen mit dem humanen Östrogenrezeptor [9]. Da Isoflavone eine hohe Affinität $\mathrm{zu}$ dem humanen ER $\beta$ Rezeptor aufweisen, können sie Östrogensignalwege aktivieren. Equol ist ein Metabolit, der von der Darmflora durch Biotransformation aus Daidzein produziert wird. Aber nicht alle Menschen, die Daidzein konsumieren, können es auch in Equol umwandeln: Nur etwa ein Drittel bis die Hälfte der Bevölkerung kann Equol im Darm produzieren [10]. In mehreren Studien konnte mit der Behandlung mit Equol eine Verbesserung der postmenopausalen Zustände gezeigt werden. Zusätzlich fand Equol eine hohe Akzeptanz bei den Patientinnen und kann daher als eine gute Behandlungsmethode für Frauen in der Menopause und besonders für jene, die keine hormonellen Präparate verwenden wollen, angesehen werden [11].

\section{Materialien und Methoden}

\section{Studiendesign}

Für den Wirksamkeitsnachweis wurde eine einarmige unkontrollierte Pilotstudie durchgeführt. Hierfür wurden entweder equolenthaltende Vaginalkapseln oder equolenthaltende Vaginalgelpräparate zur Behandlung von Vaginalatrophie bei Frauen in der Menopause verabreicht. Die Dauer der Studie betrug 16 Wochen (120 Tage).

\section{Probanden}

Für die Studie wurden 69 kaukasische Frauen rekrutiert, von denen 51 die gesamte Studie abschlossen. Einschlusskriterien für die Studie waren: postmenopausale (keine Menstruation für $12 \mathrm{Mo}$ nate, Alter über 40 Jahre mit typischen Symptomen oder follikelstimulierendes Hormon $[\mathrm{FSH}]>40 \mathrm{U} / \mathrm{l}$ bei Patientinnen, die eine Hysterektomie hatten) und zusätzlich vaginale Trockenheit, vaginaler Juckreiz, Schmerzen beim Geschlechtsverkehr oder ein vaginaler $\mathrm{pH}$-Wert $>5$ beim Screening. 


\begin{tabular}{lllll|llll}
\hline Tab. 1 & Demografische Eigenschaften der Teilnehmer der E55-RS-Studie \\
\hline Status & $\begin{array}{l}\text { Status } \\
\text { (An- } \\
\text { zahl) }\end{array}$ & $\begin{array}{l}\text { Durch- } \\
\text { schnitt- } \\
\text { licher } \\
\text { BMI }\end{array}$ & $\begin{array}{l}\text { Durch- } \\
\text { schnitts- }\end{array}$ & $\begin{array}{l}\text { Alkohol- } \\
\text { konsum }\end{array}$ & $\begin{array}{l}\text { Nikotin- } \\
\text { konsum } \\
\text { (Jahre) }\end{array}$ & $\begin{array}{l}\text { Komor- } \\
\text { bidität }\end{array}$ & $\begin{array}{l}\text { Beglei- } \\
\text { tende } \\
\text { Medi- } \\
\text { kation }\end{array}$ \\
\hline Abgeschlossen & 51 & 27,45 & 63,68 & 51 & 51 & 36 & 33 \\
\hline Abgebrochen & 11 & 27,42 & 62,13 & 11 & 11 & 8 & 7 \\
Gescreent & 7 & 29,30 & 65,67 & 7 & 7 & 6 & 5 \\
Gesamtsumme & 69 & 27,63 & 63,63 & 69 & 69 & 50 & 45
\end{tabular}

Tab. 2 BMl und Alter von Patientinnen, die die E55-RS-Studie abgeschlossen haben

\begin{tabular}{ll|llll} 
& Beobachtet & Mittelwert & $\begin{array}{l}\text { Standard- } \\
\text { fehler }\end{array}$ & 95\%-Konfidenzintervall \\
\hline BMI & 51 & 32,98 & 1,39 & 30,18 & 35,78 \\
Alter (Jahre) & 51 & 63,68 & 1,17 & 61,32 & 66,04
\end{tabular}

\begin{tabular}{lllllll}
\hline $\begin{array}{l}\text { Tab. } 3 \\
\text { Studie abge- } \\
\text { schlossen }\end{array}$ & Beobachtet & Mittelwert & $\begin{array}{l}\text { Standard- } \\
\text { abweichung }\end{array}$ & Minimum & Maximum \\
\hline Vaginaler pH 1 & 51 & 6,50 & 0,50 & 5,50 & 7,50 \\
Vaginaler pH 2 & 51 & 5,97 & 0,58 & 5,00 & 7,50 \\
Vaginaler pH 3 & 51 & 5,65 & 0,72 & 4,00 & 8,00 \\
Alter (Jahre) & 51 & 63,68 & 8,38 & 50,17 & 80,95 \\
\end{tabular}

Teilnehmer mit systemischer oder topischer Verabreichung von Östrogenen innerhalb der letzten 8 Wochen oder mit Einnahme von Kräutern mit entsprechender Wirksamkeit auf menopausale Beschwerden innerhalb von 4 Wochen wurden von der Studie ausgeschlossen, ebenso wie jene mit Vulvadystrophie, aktueller Vaginal- oder Harnwegsinfektion und jeder Vaginaloperation innerhalb von 12 Monaten oder abnormalem $\mathrm{Zy}$ tologiegrad $\geq 3 \mathrm{~b}$.

\section{Methoden und Behandlung}

Die Rekrutierung der Studienteilnehmerinnen erfolgte durch Ärzte in ausgewählten Kliniken. Potenzielle Probandinnen wurden telefonisch kontaktiert und bekamen Informationen zur Studie. Nachdem sie der Teilnahme an der Studie zugestimmt hatten, wurden sie in die Klinik eingeladen und von medizinischem Personal gescreent. Nach Einholung der Einwilligung wurde die Anamnese erhoben und ein Symptomfragebogen wurde ausgefüllt. Ein Gynäkologe führte eine körperliche Untersuchung durch. Abhängig von den Ergebnissen des Screening-Besuchs wurden alle berechtigten Personen über einen Symptomfragebogen erhoben. Informationen wie vaginales Jucken/ Jucken im Vulvabereich, vaginale Trockenheit, Schmerzen beim Geschlechtsverkehr, Symptome der Harnblase wurden abgefragt (siehe Anhang).

\section{Statistische Analyse}

Die statistische Analyse wurde mit Microsoft ${ }^{\circledR}$ Office Excel ${ }^{\circledR}$, Stata $14.1^{\circledR}$ durchgeführt und die Ergebnisse wurden als statistisch signifikant bei einem $p$ Wert $\leq 0,05$ betrachtet. T-Test, Chi-Quadrat-Test und Hypothesentest wurden durchgeführt, um mögliche Zusammenhänge zwischen Daten aus den Fragebögen und Labortests und Unterschiede zwischen der Prä- und Postintervention zu untersuchen.

\section{Ergebnisse}

\section{Demografie}

Diese Studie untersuchte 69 Patientinnen (alle Kaukasier), von denen 51 (74\%) Patientinnen eingeschlossen wurden und die Studie abschlossen, 11 Patientinnen (15\%) wurden eingeschlossen und vollendeten die Studie nicht. Das Durchschnittsalter der Patientinnen, welche die Studie abgeschlossen haben, betrug 63,68 Jahre (95\%-KI 61,32; $66,04)$, und davon hatten $29,4 \%$ einen normalen BMI. Darüber hinaus berichteten $70,5 \%$, dass sie beim Screening eine oder mehrere chronische Erkrankungen hatten. Die Daten sind in den - Tab. 1 und 2 zusammengefasst.

Der Behandlungseffekt für jede einzelne Patientin wurde ausgewertet und vom Arzt bei Besuch 2 und Besuch 3 dokumentiert. Wie in $\bullet$ Abb. 1 gezeigt, hatte E55 RS bei beiden Visiten eine insgesamt gute oder sehr gute Wirkung $(n>50)$. Die Behandlungsakzeptanz wurde von den Patientinnen bei den Visiten 2 und 3 bewertet und berichtet. Wie in 0 Abb. 2 gezeigt, wies E55 RS eine gute oder sehr gute Akzeptanz $(n>50)$ auf.

\section{Variablen von Interesse}

Änderungen in der Schwere der Symptome der vaginalen Atrophie wurden
Die Variablen von Interesse wurden für jene Patientinnen analysiert, die 


\section{Eine Proof-of-concept-Studie von Isoflavandiol-E55-RS-Vaginalkapseln oder Vaginalgel zur Linderung} der menopausalen Vaginalatrophie

\section{Zusammenfassung}

Ziel. Ziel dieser Studie war es, die Wirkung von Equol als Behandlungsmethode für Frauen mit vulvovaginaler Atrophie (VVA) in den Wechseljahren zu untersuchen.

Methoden. Für diese einarmige, unkontrollierte Pilotstudie wurden 51 klimakterische Frauen im Alter von 61 bis 64 Jahren rekrutiert. Sie erhielten genaue Anweisungen, wie sie entweder das 5cc-Lecithin-Basisgel oder die Vaginalkapseln mit jeweils $10 \mathrm{mg}$ aktivem E55 (Equol) verwenden sollten. Equol wurde in einer veganen patentierten selbstemulgierenden Kapsel (SEDD) eingekapselt. Die Dauer der Studie betrug 120 Tage (16 Wochen). Vaginale pH-Messung und vaginale mikrobiologische Tests wurden bei jeder Kontrollvisite durchgeführt. Zusätzlich wurden die Teilnehmerinnen gebeten, einen
Fragebogen auszufüllen, um die Schwere der Symptome der vaginalen Atrophie zu beurteilen.

Ergebnisse. Die Behandlung mit E55 RS (Equol) zeigte insgesamt gute Effekte und eine hohe Akzeptanz bei den Patientinnen. Symptome von VVA, wie vaginaler Juckreiz, vaginale Trockenheit und Schmerzen beim Geschlechtsverkehr, konnten verringert werden. Der mittlere vaginale $\mathrm{pH}$-Wert konnte signifikant von 6,50 auf 5,65 $(p<0,05)$ gesenkt werden. Darüber hinaus wurde eine signifikante Verschiebung der vaginalen Epithelzellen beobachtet: Oberflächliche Zellen waren erhöht (einseitig $p=0,0000$ ), während Parabasalzellen nach der Behandlung abgenommen hatten (einseitig $p=0,0001$ ). Die Anwendung von E55 RS wirkte sich auch auf die vaginale Lactobacillusspp.-Kolonisierung aus, wobei der Anteil von Frauen, die Lactobacillus-spp.-positiv waren, signifikant nach der Behandlung anstieg ( $p=0,0004)$.

Schlussfolgerung. Die Ergebnisse dieser Studie deuten darauf hin, dass die Behandlung von menopausalen vulvovaginalen Symptomen mit Vaginalkapseln, die Equol enthalten, nicht nur effektiv und gut verträglich ist, sondern auch die Lebensqualität von Frauen in der Menopause verbessern kann.

Schlüsselwörter

Menopause - Vaginale Atrophie - Vaginale Kapseln · Equol · Phytoöstrogene

\section{Proof-of-Concept Study of Isoflavandiol E55-RS Vaginal Capsules or Vaginal Gel for the Alleviation of Menopausal Vaginal Atrophy}

Abstract

Aim. The aim of this study was to investigate the efficacy of equol in the treatment of menopausal vulvovaginal atrophy (VVA). Methods. For this single-arm, uncontrolled pilot study, 51 postmenopausal women aged 61-64 years were recruited. They received precise instructions on the use of either the $5 c c$ lecithin basis gel or vaginal capsules with $10 \mathrm{mg}$ active E55 (equol) each. Equol was encapsulated in a vegan, patented, selfemulsifying drug delivery (SEDD) capsule. The duration of the study was 120 days (16 weeks) Vaginal $\mathrm{pH}$ measurements and vaginal microbiological tests were performed at each follow-up visit. In addition, the participants were asked to complete a questionnaire to assess the severity of the vaginal atrophy symptoms.

Results. Treatment with E55 RS (equol) showed overall good efficacy and high acceptance in the patients. Symptoms of VVA, such as vaginal itching, vaginal dryness and pain during intercourse, were reduced. The average vaginal $\mathrm{pH}$ could be lowered significantly from 6.50 to $5.65(p<0.05)$. In addition, a significant shift of vaginal epithelial cells was observed: superficial cells increased (one-tailed $p=0.0000$ ), while parabasal cells decreased after treatment (one-tailed $p=0.0001$ ). The use of E55 RS also affected vaginal lactobacillus spp. colonization, with the proportion of women who tested positive for lactobacillus spp. ( $p=0.0004)$ significantly increasing after treatment.

Conclusion. The results of this study suggest that treatment of menopausal vulvovaginal symptoms with vaginal capsules containing equol is not only effective and well-tolerated, but may also improve the quality of life of menopausal women.

\section{Keywords}

Menopause - Vaginal atrophy · Vaginal capsules · Equol · Phytoestrogen die Studie vom Screening bis zur Beendigung durchführten $(n=51)$. Diese Variablen umfassten: vaginaler $\mathrm{pH}$-Wert, vaginale Epithelzellen und von der $\mathrm{Pa}$ tientin berichtete Symptome (vaginale Trockenheit, Dyspareunie/Schmerzen während des Geschlechtsverkehrs, vaginaler/vulvaler Juckreiz und Symptome der Harnblase). Die Analyse berücksichtigte die im Studienprotokoll vorgeschlagenen Hypothesen. In der Gruppe von 51 Patientinnen - die die Studie abgeschlossen hatten - fehlten keine Daten zu den Variablen von Interesse.

Einige der Gründe für den Studienabbruch waren: eigener Wille, Hautausschlag, Unzufriedenheit mit dem Produkt und akute Niereninfektion.

\section{Vaginaler $\mathrm{pH}$ wurde bei jedem der drei Besuche gemessen}

Diese Studie stellte die Hypothese auf, dass die Anwendung des E55 RS den vaginalen $\mathrm{pH}$-Wert der Patientinnen sen- ken würde. Ein Student-t-Test für gepaarte Proben mit 95\%-KI wurde durchgeführt, um die durchschnittlichen $\mathrm{pH}$ Werte zwischen den einzelnen Besuchen $\mathrm{zu}$ vergleichen. In $\bullet$ Tab. 3 werden die Ergebnisse des mittleren $\mathrm{pH}$-Werts, welcher bei jedem Besuch gemessen wurde, dargestellt.

Die in - Tab. 3 gezeigten Ergebnisse stützen die alternative Hypothese (Ha: Mittelwert (diff) $>0$ ), dass der vaginale $\mathrm{pH}$ bei der dritten Visite niedriger ist (ver- 


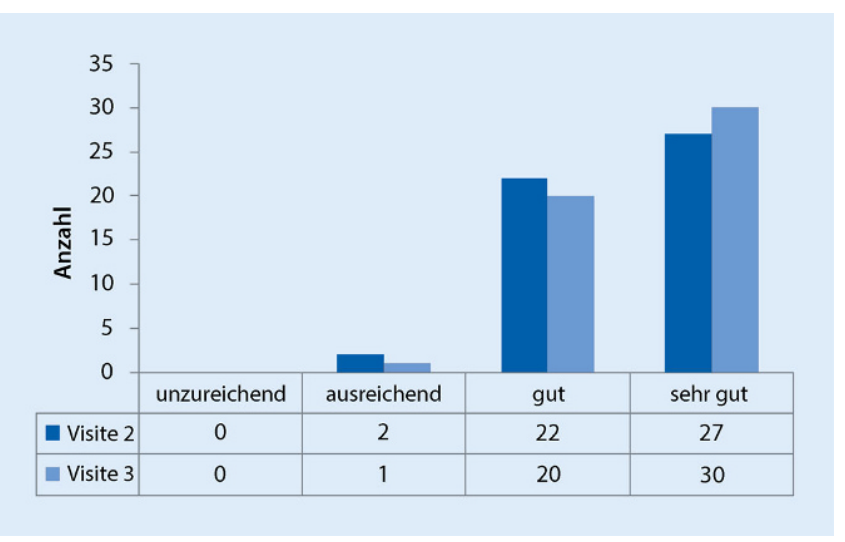

Abb. 1 \& Übersicht des Behandlungseffekts von E55 RS

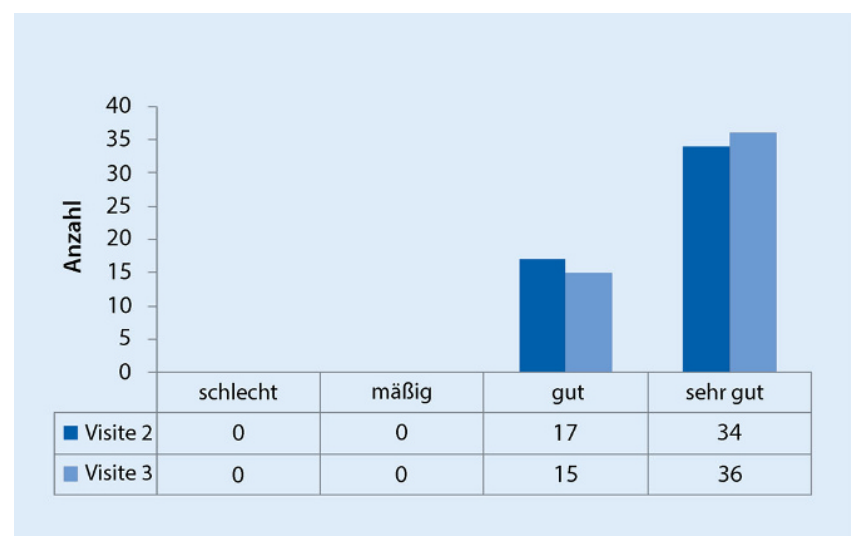

Abb. $2 \triangle$ Akzeptanz der Behandlung mit E55 RS bei den Patientinnen

Tab. 4 Reifegrad („maturation value " [MV]) und Anteil der drei Arten von vaginalen Epithelzellen vor und nach Behandlung mit E55 RS

\begin{tabular}{|c|c|c|c|c|c|c|c|c|c|}
\hline \multirow{2}{*}{$\begin{array}{l}\text { Patientinnen, die die } \\
\text { Studie abgeschlossen } \\
\text { haben }\end{array}$} & \multicolumn{3}{|l|}{$\mathbf{M V}^{\mathrm{a}}$} & \multicolumn{3}{|c|}{ Epithelzellen vor Behandlung ${ }^{b}$} & \multicolumn{3}{|c|}{ Epithelzellen nach Behandlung } \\
\hline & MV1 & MV2 & $\begin{array}{l}\text { Differenz } \\
\text { M2-M1 }\end{array}$ & $\begin{array}{l}\text { Para- } \\
\text { basal } 1\end{array}$ & $\begin{array}{l}\text { Inter- } \\
\text { mediär } 1\end{array}$ & $\begin{array}{l}\text { Super- } \\
\text { fizial } 1\end{array}$ & $\begin{array}{l}\text { Para- } \\
\text { basal } 2\end{array}$ & $\begin{array}{l}\text { Inter- } \\
\text { mediär } 2\end{array}$ & $\begin{array}{l}\text { Super- } \\
\text { fizial } 2\end{array}$ \\
\hline Mittelwert & 0,32 & 0,55 & 0,22 & 0,45 & 0,46 & 0,10 & 0,09 & 0,72 & 0,19 \\
\hline Standardfehler & 0,04 & 0,03 & 0,04 & 0,06 & 0,05 & 0,02 & 0,03 & 0,04 & 0,03 \\
\hline Median & 0,35 & 0,55 & 0,13 & 0,30 & 0,50 & 0,00 & 0,00 & 0,80 & 0,10 \\
\hline Modus & 0,55 & 0,55 & 0,00 & 0,00 & 0,10 & 0,00 & 0,00 & 0,90 & 0,10 \\
\hline Standardabweichung & 0,25 & 0,18 & 0,25 & 0,40 & 0,34 & 0,17 & 0,22 & 0,28 & 0,24 \\
\hline Stichprobenvarianz & 0,06 & 0,03 & 0,06 & 0,16 & 0,12 & 0,03 & 0,05 & 0,08 & 0,06 \\
\hline Wölbung & $-1,09$ & 2,85 & $-0,68$ & $-1,81$ & $-1,70$ & 10,11 & 9,17 & 0,90 & 4,05 \\
\hline Schiefe & 0,27 & $-0,50$ & 0,45 & 0,13 & 0,04 & 2,86 & 3,15 & $-1,44$ & 2,15 \\
\hline Spannweite & 0,95 & 0,95 & 1,13 & 1,00 & 1,00 & 0,90 & 0,95 & 0,95 & 0,95 \\
\hline Minimum & 0,00 & 0,03 & $-0,23$ & 0,00 & 0,00 & 0,00 & 0,00 & 0,05 & 0,00 \\
\hline Maximum & 0,95 & 0,98 & 0,90 & 1,00 & 1,00 & 0,90 & 0,95 & 1,00 & 0,95 \\
\hline Summe & 16,48 & 27,91 & 11,44 & 22,90 & 23,25 & 4,85 & 4,74 & 36,70 & 9,56 \\
\hline Anzahl & 51 & 51 & 51 & 51 & 51 & 51 & 51 & 51 & 51 \\
\hline $95 \%$-Konfidenzintervall & 0,07 & 0,05 & 0,07 & 0,11 & 0,10 & 0,05 & 0,06 & 0,08 & 0,07 \\
\hline
\end{tabular}

glichen mit der zweiten, $p<0,05$, und der ersten Visite, $p<0,05$; $\bullet$ Abb. 3 ), der mittlere vaginale $\mathrm{pH}$-Wert ist bei der zweiten Visite niedriger (im Vergleich zur ersten Visite $p<0,05)$.

\section{Reifegrad (MV) und vaginale Epithelzellen}

Proben des Vaginalepithels wurden bei dem ersten und zweiten Besuch entnommen und untersucht (ausführendes Labor: Synlab Pharma-Institut, Bayerstr. 53, 80335 München, Deutschland). • Tab. 4 zeigt eine Zusammenfassung des Reifungswerts und der Auszählungen aller Arten von vaginalen Epithelzellen (dar- gestellt in \%), vor und nach der Behandlung mit E55 RS. Der Reifungswert wird berechnet als: 0,0 * Parabasalzellen (\%) $+0,5 *$ Intermediärzellen $(\%)+1,0$ * Superfizialzellen (\%), während der Prozentsatz der Epithelzellen auf der Basis von 200 gezählten Zellen berechnet wird. Ein erhöhter Reifegrad sowie ein höherer Prozentsatz an superfizialen und intermediären Zellen kann nach der Behandlung beobachtet werden. Entsprechend war der Anteil der Parabasalzellen bei der dritten Visite geringer. Der mittlere Prozentsatz an Epithelzellen, die innerhalb der Gruppe vor und nach der Behand- lung gemessen wurden, ist in - Abb. 4 gezeigt.

Die Studie stellte die Hypothese auf, dass die Behandlung mit E55 RS zu einer Abnahme des Anteils der Parabasalzellen und einer Zunahme der superfizialen Zellen führen kann. Da die Werte der parabasalen und superfizialen Zellen von Besuch 1 und 3 nicht normal verteilt waren (Shapiro-Wilk: parabasale Zellen prä: W 0,85651, $p=0,00002$, post: W 0,53640, $p=0,00000$; superfiziale Zellen prä W 0,69628, $p=0,00000$ und post W 0,69952, $p=0,00000$ ), wurde ein Sign-Test durchgeführt, um die Ergebnisse der beiden Messungen zu vergleichen. 


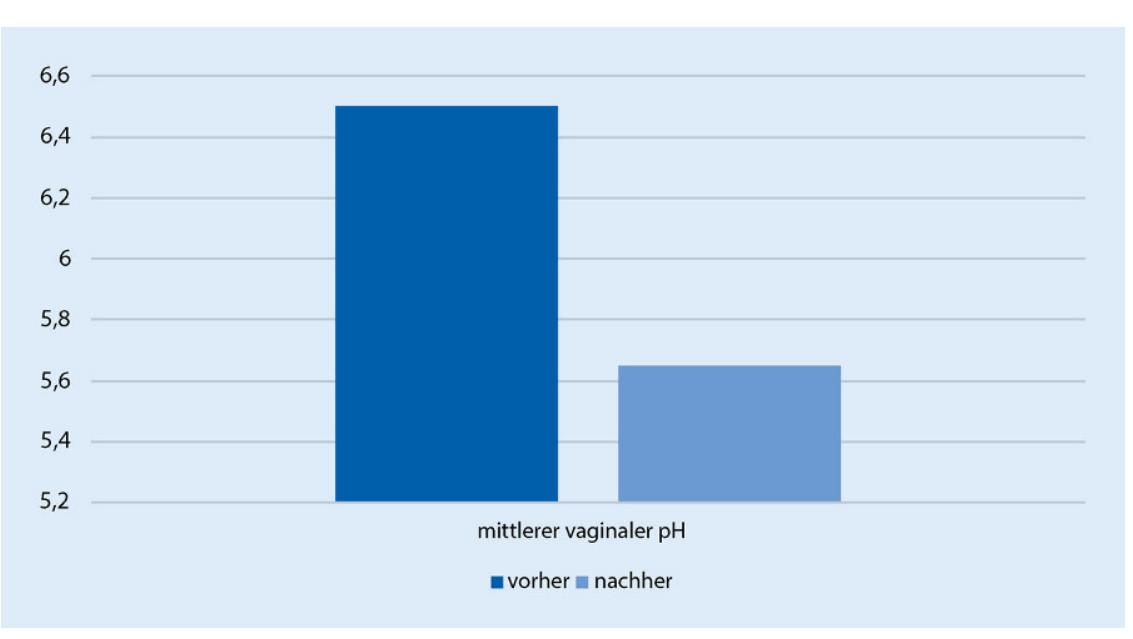

Abb. 3 × Veränderung des vaginalen pH-Werts vor und nach der Behandlung mit E55 RS

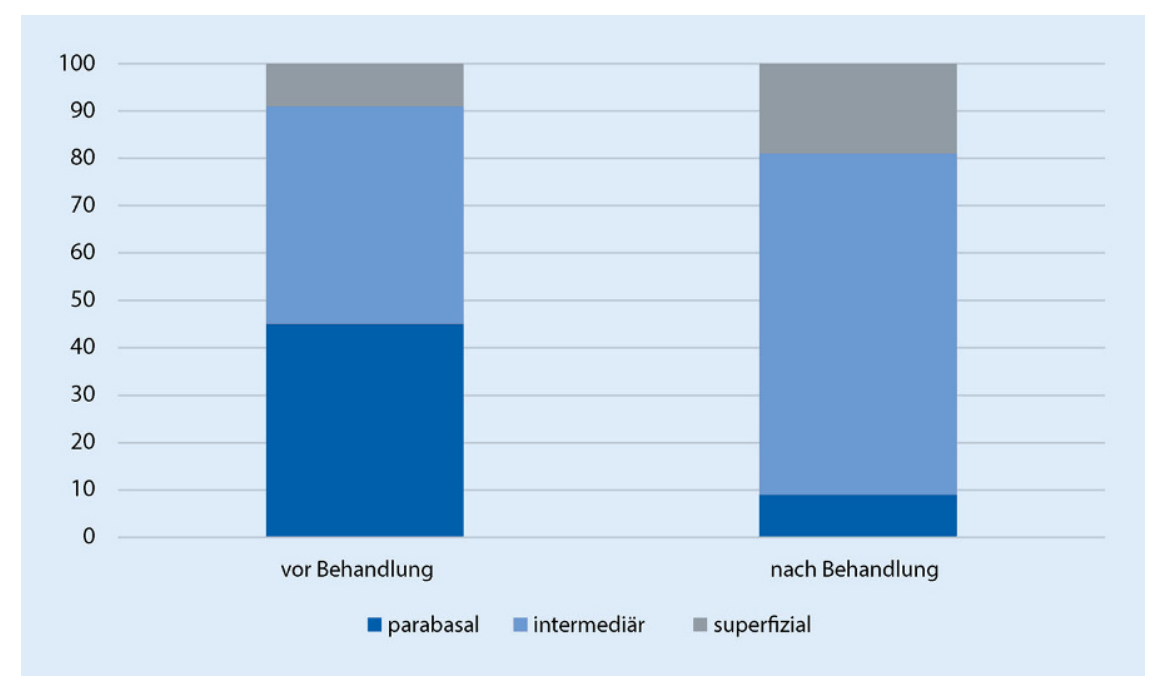

Abb. 4 A Mittlere Anzahl an vaginalen Epithelzellen vor und nach der Behandlung mit E55 RS (in Prozent)

Die Ergebnisse bei beiden Zelltypen erlauben die Ablehnung der Ho (bei einem Signifikanzniveau von 0,05 ) für die Gleichheit des Medianunterschieds zwischen den prä und post gemessenen Prozentsätzen und folgern, dass der mediane prozentuale Anteil der vaginalen Parabasalzellen höher ist $(p=0,0001)$, während der mediane prozentuale Anteil der vaginalen superfizialen Zellen mit E55 RS höher ist (einseitig $p=0,0000$ ). Die Testergebnisse sind in $\bullet$ Tab. 4 dargestellt. ten aus den Fragebögen wurden als Likert-Typ behandelt und jedes Symptom wurde separat analysiert. Eine Zusammenfassung für alle vier Symptome ist in - Tab. 5 und $-A b b .5$ dargestellt.

Zusätzlich wurden die Daten so transformiert, dass Fragebögen, die eine negative Veränderung ausdrücken (z.B. von 5 auf 1 geändert), als „verbessert“, solche mit einer Differenz von null als „unverändert" und solche mit positiver Veränderung (z.B. von 1 nach 4 ) als „verschlechtert" vermerkt wurden. Anschließend wurden die Gruppen „unverändert" und „verschlechtert" in einer neuen Gruppe zusammengefasst - „nicht verbessert“. Wie in diesen Abbildungen gezeigt wird, konnte eine deutliche Verbesserung der vaginalen Trockenheit nach der Behandlung erzielt werden (98,4\%). Der Grad der Besserung der übrigen Symptome, die bei der Visite 3 verzeichnet wurden, war etwas geringer im Vergleich zum Beginn der Intervention (Besuch 1).

Die Studie stellte die Hypothese auf, dass die Behandlung mit E55 RS zu einer Verbesserung der vaginalen Trockenheit, des vaginalen Juckreizes oder der Dyspareunie (Schmerzen beim Geschlechtsverkehr) führen kann. Um zu testen, ob der Schweregrad der selbst berichteten Symptome zwischen den Visiten variiert, wurden paarweise Antworten als Ordinalskala ( 1 am wenigsten schwerwiegend, 5 am schwersten) unter Annahme nicht normalverteilter Daten analysiert (Shapiro-Wilk-W-Test stützte die Annahme für mehr als $60 \%$ der Artikel). Vergleiche des berichteten Schweregrads wurden innerhalb jedes der folgenden Sets gemacht: Visite 1 und 2, Visite 2 und 3 und Visite 1 und 3. Die Ergebnisse der statistischen Tests ermöglichen die Ablehnung der Nullhypothese bei einem Signifikanzniveau von 0,05 und lassen daraus schlussfolgern, dass die Mediandifferenzen nicht gleich 0 sind. Da die Unterschiede positiv sind, kann geschlussfolgert werden, dass für alle genannten Symptome der Schweregrad bei jedem Folgebesuch verringert werden konnte. 


\begin{tabular}{|c|c|c|c|c|c|c|c|c|c|}
\hline & Häufigkeit & Anteil (\%) & Kumulativ & Häufigkeit & Anteil (\%) & Kumulativ & Häufigkeit & Anteil (\%) & Kumulativ \\
\hline Vaginale Trockenheit & Visite 1 & & & Visite 2 & & & Visite 3 & & \\
\hline Nein & 1 & 1,96 & 1,96 & 2 & 3,92 & 3,92 & 28 & 54,9 & 54,9 \\
\hline Sehr leicht & 2 & 3,92 & 5,88 & 14 & 27,45 & 31,37 & 20 & 39,22 & 94,12 \\
\hline Leicht & 17 & 33,33 & 39,22 & 29 & 56,86 & 88,24 & 3 & 5,88 & 100 \\
\hline Erhöht & 30 & 58,82 & 98,04 & 6 & 11,76 & 100 & - & - & - \\
\hline Stark & 1 & 1,96 & 100 & - & - & - & - & - & - \\
\hline Gesamt & 51 & 100 & - & 51 & 100 & - & 51 & 100 & - \\
\hline $\begin{array}{l}\text { Schmerz bei Geschlechtsver- } \\
\text { kehr }\end{array}$ & Visite 1 & & & Visite 2 & & & Visite 3 & & \\
\hline Nein & 24 & 47,06 & 47,06 & 26 & 50,98 & 50,98 & 39 & 76,47 & 76,47 \\
\hline Sehr leicht & 6 & 11,76 & 58,82 & 11 & 21,57 & 72,55 & 8 & 15,69 & 92,16 \\
\hline Leicht & 9 & 17,65 & 76,47 & 13 & 25,49 & 98,04 & 3 & 5,88 & 98,04 \\
\hline Erhöht & 11 & 21,57 & 98,04 & 1 & 1,96 & 100 & 1 & 1,96 & 100 \\
\hline Stark & 1 & 1,96 & 100 & - & - & - & - & - & - \\
\hline Gesamt & 51 & 100 & - & 51 & 100 & - & 51 & 100 & - \\
\hline Vaginaler Juckreiz & Visite 1 & & & Visite 2 & & & Visite 3 & & \\
\hline Nein & 17 & 33,33 & 33,33 & 30 & 58,82 & 58,82 & 41 & 80,39 & 80,39 \\
\hline Sehr leicht & 14 & 27,45 & 60,78 & 14 & 27,45 & 86,27 & 7 & 13,73 & 94,12 \\
\hline Leicht & 9 & 17,65 & 78,43 & 6 & 11,76 & 98,04 & 3 & 5,88 & 100 \\
\hline Erhöht & 11 & 21,57 & 100 & 1 & 1,96 & 100 & - & - & - \\
\hline Stark & - & - & - & - & - & - & - & - & - \\
\hline Gesamt & 51 & 100 & - & 51 & 100 & - & 51 & 100 & - \\
\hline Urinale Symptome & Visite 1 & & & Visite 2 & & & Visite 3 & & \\
\hline Nein & 16 & 31,37 & 31,37 & 29 & 56,86 & 56,86 & 36 & 70,59 & 70,59 \\
\hline Sehr leicht & 16 & 31,37 & 62,75 & 10 & 19,61 & 76,47 & 8 & 15,69 & 86,27 \\
\hline Leicht & 11 & 21,57 & 84,31 & 8 & 15,69 & 92,16 & 6 & 11,76 & 98,04 \\
\hline Erhöht & 8 & 15,69 & 100 & 4 & 7,84 & 100 & 1 & 1,96 & 100 \\
\hline Stark & - & - & - & - & - & - & - & - & - \\
\hline Gesamt & 51 & 100 & - & 51 & 100 & - & 51 & 100 & - \\
\hline
\end{tabular}

\section{Lactobacillus spp. in der vaginalen Mikroflora}

Mikrobiologische Tests (Vaginalabstriche) wurden zu Beginn der Studie (Visite 1) und nach Abschluss der Behandlung mit E55 RS (Visite 3) durchgeführt. Die Ergebnisse sind in $\bullet$ Tab. 6 und $\bullet$ Abb. 6 dargestellt. Insgesamt ist der Anteil an Patientinnen, die bei Besuch 1 Lactobacillus-spp.-positiv waren $13,73 \%$, im Vergleich zu 86,27\%, deren vaginales Mikrobiologieergebnis negativ war. Bei Besuch 3 veränderten sich diese Anteile auf 45,1\% (3,3-mal erhöht) bzw. 54,9\% (1,57-fach verringert).

Es wird keine Änderung gegenüber den Ausgangsergebnissen angenommen, d.h. die Anteile von Lactobacillus-spp.positiven oder -negativen Proben unterschieden sich zwischen Visite 1 und
Visite 3 nicht. Der paarweise Vergleich der Messungen vor und nach der Behandlung zeigte jedoch, dass 18 (35\%) der Patientinnen bei der letzten Visite eine Verbesserung dieses mikrobiologischen Kriteriums aufwiesen, während 2 (3,8\%) ein schlechteres Ergebnis hatten. Von den 31 Patientinnen, deren Status sich nicht änderte, blieben nur 5 (9,8\%) positiv für Lactobacillus spp. bei Besuch 3 . Die Durchführung des McNemar-Tests (1 df), $p=0,0004$, belegt, dass die Änderung des Anteils der Lactobacillus-spp.positiven Proben, nach der Behandlung statistisch signifikant ist.

\section{Diskussion}

Die Studie zeigt, dass E55 RS eine sehr gute nichthormonelle Behandlungsvariante für menopausale Frauen mit vulvovaginaler Atrophie ist, da es drei sehr wichtige Ergebnisse erzielte: Es vermindert den vaginalen $\mathrm{pH}$-Wert effizient, vaginale oberflächliche Zellen werden erhöht und die Schwere der vaginalen Trockenheit wird reduziert. Da die normale, gesunde Vaginalflora von Lactobacillus spp. dominiert wird, hat eine Veränderung ihrer Anzahl mehrere Konsequenzen. Im Falle der Menopause wird eine Reduktion des vaginalen Lactobacillus spp. beobachtet, was zu einerverminderten Pathogenabwehr und darüber hinaus zur potenziellen Entwicklung von bakterieller Vaginose führt [12]. Nach der Behandlung mit E55 RS stieg die Anzahl der Patientinnen mit vaginaler Lactobacillus-spp.-Kolonisation von 7 auf 13 Patientinnen an, was darauf hindeutet, dass Equol einen positiven Einfluss auf 


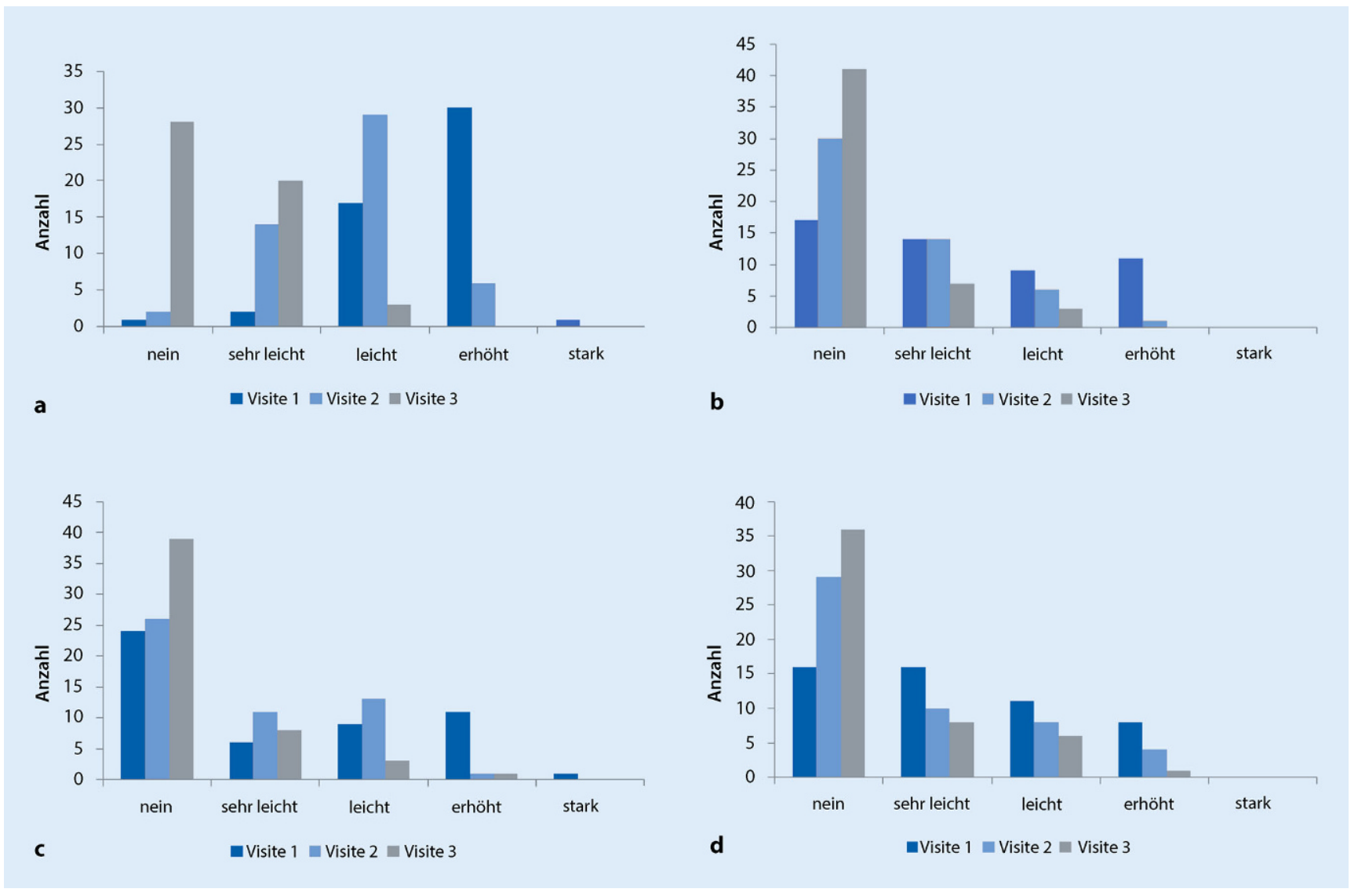

Abb. 5 \& a Symptomfragebogen, vaginale Trockenheit, b Symptomfragebogen, vaginales Jucken, c Symptomfragebogen, Schmerzen beim Geschlechtsverkehr, d Symptomfragebogen, urinale Symptome

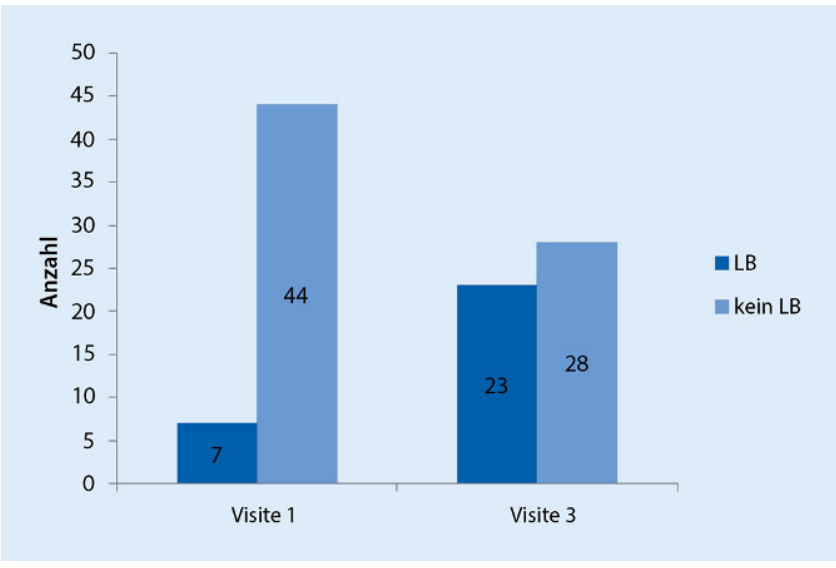

Abb. $6<$ Vaginale Mikrobiologie: Patientinnen mit Lactobacillus spp. (LB) vor und nach der Behandlung mit E55 RS

die bakterielle Rekolonisation hat. Andere Studien haben gezeigt, dass Lactobacillus spp. bei postmenopausalen Frauen im Vergleich zu prämenopausalen verringert ist, was auf den verringerten Östrogenspiegel zurückzuführen ist [13]. Darüber hinaus führt die postmenopausale Reduktion der Lactobacillus-spp.-Kolonisierung aufgrund der verminderten Produktion von Milchsäure zu einem An- stieg des vaginalen $\mathrm{pH}$-Werts. Folglich ist der $\mathrm{pH}$ bei postmenopausalen Frauen erhöht ( $\mathrm{pH}=6$, verglichen mit $\mathrm{pH}=4,5$ bei prämenopausalen Frauen), was zu einem höheren Risiko einer bakteriellen Infiltration der Vagina führt [12]. Die Behandlung mit E55 senkte den $\mathrm{pH}$-Wert der Patientinnen signifikant. Verglichen mit anderen Studien, die mit verschiedenen Östradiolpräparaten durchgeführt wurden, konnte E55 RS eine deutlich höhere Abnahme des $\mathrm{pH}$ erzielen $[14,15]$. Dieser Effekt wurde auch in anderen Studien mit Equolpräparaten zur VVA-Behandlung [8] dokumentiert und legt nahe, dass die Behandlung mit Equol im Vergleich zu Östradiol einen Vorteil bei der Senkung des $\mathrm{pH}$-Werts hat.

Darüber hinaus konnte die Behandlung mit E55 RS eine Veränderung der vaginalen Epithelzellzusammensetzung erreichen. Da der Östrogenverlust nach der Menopause zu einer ungünstigen Veränderung der Vaginalzellen führt, indem oberflächliche Zellen vermindert sind und Parabasalzellen zunehmen, ist der beobachtete Effekt von E55 RS sehr günstig und wünschenswert. Im Vergleich zur vaginalen Östradiolapplikation konnte E55 die oberflächlichen Zellen um einen höheren Prozentsatz erhöhen und konnte auch die Anzahl der parabasalen Zellen verringern [15].

Wenn es um VVA-Symptome wie vaginale Trockenheit, vaginalen Juck- 
Tab. 6 Vaginalermikrobiologischer Befund, Patientinnen mit positivem Lactobacillus-spp.-Befund vor und nach Behandlung mit E55 RS

\begin{tabular}{l|lllll} 
& Vorher & & Nachher & \\
& Häufigkeit & Anteil (\%) & Häufigkeit & Anteil (\%) \\
\hline LB & 7 & 13,73 & 23 & 45,1 \\
Kein LB & 44 & 86,27 & 28 & 54,9 \\
\hline Gesamt & 51 & 100 & 51 & 100
\end{tabular}

reiz oder Schmerzen während des Geschlechtsverkehrs geht, könnte die Behandlung mit E55 RS den Schweregrad von allen genannten Symptomen verbessern. Da etwa $50 \%$ der postmenopausalen Frauen an VVA leiden, ist die Behandlung dieser Symptome wichtig, um ihre Lebensqualität zu verbessern.

Die Verbesserung der Wirkung von Phytoöstrogenen auf Wechseljahresbeschwerden wurde auch in anderen Studien berichtet, und da sie das Brustkrebsrisiko nicht erhöhen, haben sie einen großen Vorteil gegenüber der Hormonersatztherapie (HRT; [11, 16, 17]). Verschiedene Studien haben gezeigt, dass die HRT, insbesondere eine Kombination aus Östrogen und Progesteron, das Risiko für Brust-, Endometriumund Herz-Kreislauf-Erkrankungen erhöht [18-21]. Darüber hinaus ist die Phytoöstrogentherapie eine geeignete Behandlungsmethode für Frauen in der Menopause, die an Brustkrebs leiden, da der Einsatz der HRT in dieser Patientengruppe nach wie vor kontrovers diskutiert wird [22].

Eine Stärke dieser Studie ist die Verwendung von veganen selbstemulgierenden Kapseln. Insgesamt zeigte die Behandlung mit E55 RS eine sehr hohe Akzeptanz und gute Ergebnisse.

\section{Schlussfolgerung}

Die Behandlung von Symptomen der vaginalen Atrophie mit E55 RS bei postmenopausalen Frauen war insgesamt sehr gut und das Präparat wurde von den $\mathrm{Pa}$ tientinnen gut angenommen. Es kann eine Alternative für Frauen mit vaginaler Atrophie sein, die sich keiner Hormontherapie unterziehen wollen, aber besonders für Frauen mit Brustkrebs, bei denen eine HRT kontraindiziert ist.

\section{Korrespondenzadresse}

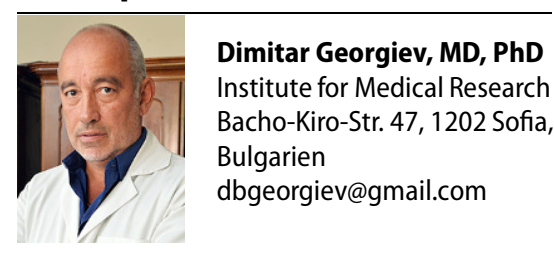

Danksagung. Die Autoren danken der Firma System Biologie AG, Wollerau, Schweiz, für die Zurverfügungstellung des Präparats Isoflavandiol E55-RS Vaginalkapseln bzw. Vaginalgel. Ein besonderer Dank gilt Herrn Prof DDr. Huber für seine langjährige Mitarbeit im Bereich Equolforschung und für seine Mithilfe im Aufbau des Studiendesigns.

\section{Einhaltung ethischer Richtlinien}

Interessenkonflikt. L. Mayr, D. Georgievund A. Toulev geben an, dass kein Interessenkonflikt besteht.

Alle beschriebenen Untersuchungen am Menschen wurden mit Zustimmung der zuständigen Ethik-Kommission, im Einklang mit nationalem Recht sowie gemäß der Deklaration von Helsinki von 1975 (in der aktuellen, überarbeiteten Fassung) durchgeführt. Von allen beteiligten Patienten liegt eine Einverständniserklärung vor. 


\section{Anhang A}

\section{A.1. Symptom questionnaire} (complete at each study visit)

Please read carefully and answer each question by checking the box that applies with „X“ (• Tab. 7).

\section{A.2. Assessment of Therapeutic} Success (Doctor) (Only V2 and V3)

Very Good: $\square$; Good: $\square$; Sufficient: $\square$; Not enough: $\square$.

\section{A.3. Adverse Effects}

Is the patient complaining about adverse effects: no: $\square ;$ yes: $\square$. (please fill in below ${ }^{\star}$ )
Tab. 7 Symptom Questionnaire

Symptoms

No

Very light Light

Expressed Strongly

Symptomatology before or after treatment with E55 Comfort \& Dryness ( $10 \mathrm{mg}$ equol)

Visit Nr.

Visit date:

Date of treatment start:

Vaginal dryness $^{\mathrm{a}}$

Pain during sexual

intercourse

Vaginal itching

Urinary symptoms $^{\mathrm{d}}$

\begin{tabular}{l|l}
\hline$\square$ & $\square$ \\
$\square$ & $\square$ \\
$\square$ & $\square$ \\
$\square$ & $\square$
\end{tabular}

$$
\begin{aligned}
& \text {.................... (dd/mm/yyyy) } \\
& \text {.................... (dd/mm/yyyy) }
\end{aligned}
$$$$
\square \quad \square \quad \square
$$$$
\square \quad \square \quad \square
$$

aSexual intercourse without a lubricant is sore and painful (many women report "dry and uncomfortable" rather than sore and painful which is still clinically significant). There are minimal secretions on underwear

${ }^{\text {b}}$ Sex is painful, dry and sore, either during intercourse or within $10 \mathrm{~min}$. There is a need to use lubricants

"There is discomfort caused by underwear. The skin feels like „sand-paper". Need to use a product to reduce the vaginal itching

dUrinary frequency, strong urge to urinate, incontinence

\section{Remarks*}

Hier steht eine Anzeige.

\section{Springer}


Open Access. Dieser Artikel wird unter der Creative Commons Namensnennung 4.0 International Lizenz (http://creativecommons.org/licenses/by/4.0/deed. de) veröffentlicht, welche die Nutzung, Vervielfältigung, Bearbeitung, Verbreitung und Wiedergabe in jeglichem Medium und Format erlaubt, sofern Sie den/die ursprünglichen Autor(en) und die Quelle ordnungsgemäß nennen, einen Link zur Creative Commons Lizenz beifügen und angeben, ob Änderungen vorgenommen wurden

Hinweis des Verlags. Der Verlag bleibt in Hinblick auf geografische Zuordnungen und Gebietsbezeichnungen in veröffentlichten Karten und Institutsadressen neutral.

\section{Literatur}

1. Farrell EAM (2017) Genitourinary syndrome of menopause. Aust Fam Physician 46(7):481-484. https://doi.org/10.1097/GME. 0000000000000329

2. Monteleone P, Mascagni G, Giannini A, Genazzani AR, Simoncini T (2018) Symptoms of menopause-Global prevalence, physiology and implications. Nat Rev Endocrinol 14(4):199-215. https://doi.org/10.1038/nrendo.2017.180

3. Repše-Fokter A, Takač I, Fokter SK (2008) Postmenopausal vaginal atrophy correlates with decreased estradiol and body mass index and does not depend on the time since menopause. Gynecol Endocrinol 24(7):399-404. https://doi. org/10.1080/09513590802217290

4. Mueck AO, Ruan X, Prasauskas V, Grob P, Ortmann $O$ (2018) Treatment of vaginal atrophy with estriol and lactobacilli combination: a clinical review. Climacteric. https://doi.org/10.1080/13697137. 2017.1421923

5. Nappi RE, Gardella B (2015) Vulvo-vaginal atrophy In: Manag menopause 21st century solut, S 52-57 https://doi.org/10.1017/CB09781316091821.009

6. Sturdee DW, Panay N (2010) Recommendations for the management of postmenopausal vaginal atrophy. Climacteric 13(6):509-522. https://doi. org/10.3109/13697137.2010.522875

7. Nilsson K, Risberg B, Heimer G (1995) The vaginal epithelium in the postmenopause-cytology, histology and $\mathrm{pH}$ as methods of assessment Maturitas 21(1):51-56. https://doi.org/10.1016/ 0378-5122(94)00863-3

8. Caruso S, Cianci S, Fava V, Rapisarda AMC, Cutello S, Cianci A (2018) Vaginal health of postmenopausal women on nutraceutical containing equol. Menopause 25(4):1. https://doi.org/10.1097/GME. 0000000000001061

9. Pilsková L, Riecanskýl, Jagla F (2010) The physiological actions of isoflavone phytoestrogens. Physiol Res 59(5):651-664

10. Yuan JP, Wang JH, Liu X (2007) Metabolism of dietary soy isoflavones to equol by human intestinal microflora-Implications for health. Mol Nutr Food Res 51(7):765-781. https://doi.org/10. 1002/mnfr.200600262

11. Davinelli S, Scapagnini G, Marzatico F, Nobile V, Ferrara N, Corbi G (2017) Influence of equol and resveratrol supplementation on healthrelated quality of life in menopausal women: a randomized, placebo-controlled study. Maturitas 96:77-83. https://doi.org/10.1016/j.maturitas. 2016.11.016

12. Petricevic L, Domig KJ, Nierscher FJ et al (2013) Differences in the vaginal lactobacilli of postmeno- pausal women and influence of rectal lactobacilli. Climacteric 16(3):356-361. https://doi.org/10. 3109/13697137.2012.725788

13. Brotman R, Shardell MGP, Fadrosh D, Chang K Silver MVR, Burke A, Ravel JGP (2014) Association between the vaginal microbiota, menopause status and signs of vulvovaginal atrophy. Menopause 21(5):450-458. https://doi.org/10.1097/ GME.0b013e3182a4690b

14. Pickar JH, Amadio JM, Hill JM, Bernick BA, Mirkin S (2016) A randomized, double-blind, placebocontrolled phase 2 pilot trial evaluating a novel, vaginal softgel capsule containing solubilized estradiol. Menopause 23(5):506-510. https://doi. org/10.1097/GME.0000000000000592

15. ArcherDF, Kimble TD, Lin FDY, Battucci S, Sniukiene V, Liu JH (2017) A randomized, multicenter, double-blind, study to evaluate the safety and efficacy of estradiol vaginal cream $0.003 \%$ in postmenopausal women with vaginal dryness as the most bothersome symptom. J Womens Heal. https://doi.org/10.1089/jwh.2017.6515

16. Utian WH, Jones M, Setchell KDR (2015) S-equol: a potential nonhormonal agent for menopause-related symptom relief. J Womens Heal 24(3):200-208. https://doi.org/10.1089/jwh.2014. 5006

17. Ahsan M, Mallick AK (2017) The effect of soy isoflavones on the menopause rating scale scoring in perimenopausal and postmenopausal women: a pilot study. J Clin Diagn Res 11(9):FC13-FC16. https://doi.org/10.7860/JCDR/2017/26034.10654

18. Stute P, Wildt L, Neulen J (2018) The impact of micronized progesterone on breast cancer risk: a systematic review. Climacteric 21(2):111-122. https://doi.org/10.1080/13697137.2017.1421925

19. Tavani A, La Vecchia C (1999) The adverse effects of hormone replacement therapy. Drugs Aging 14(5):347-357

20. Flesch-Janys $D$, Slanger $T$, Mutschelknauss $E$ et al (2008) Risk of different histological types of postmenopausal breast cancer by type and regimen of menopausal hormone therapy. Int J Cancer 123(4):933-941. https://doi.org/10.1002/ ijc. 23655

21. Shah NR, Borenstein JJ, Dubois RW (2015) Postmenopausal hormone therapy and breast cancer: a systematic review and meta-analysis. Menopause 25(8):713-724. https://doi.org/10. 1097/MCA.0000000000000178.Endothelial

22. Oyarzún MFG, Castelo-Branco C (2017) Local hormone therapy for genitourinary syndrome of menopause in breast cancer patients: is it safe? Gynecol Endocrinol 33(6):418-420. https://doi. org/10.1080/09513590.2017.1290076
23. JAHRESTAGUNG 8,-9.11.2019

\section{SAVE the DATE}

"PERIMENOPAUSALE MEDIZIN ZIELGERICHTET UND RISIKOADAPTIERT"

\section{Jahrestagung Deutsche Menopause Gesellschaft e.V.}

„Perimenopausale Medizin zielgerichtet und Risiko-adpatiert"

\section{Workshops}

- Was sollten Frauenärzte/Innen von der Inneren Medizin wissen?

- Sexualmedizin in der frauenärztlichen Praxis

- Laboranalytik für Frauenärzte

- Osteoporose in der gynäkologischen Praxis

\section{Sessions}

- Was hilft bei welchen Beschwerden

- Knochen und Gelenke

- HOT TOPICS - neue Medikamente und Digitalisierung der Medizin

- Pro- und Contra-Sitzungen: „Blutungsstörungen in der Peri- und Postmenopause..." und „HRT - transdermal versus oral"

- Berichte u. a. zu Fundraising für Frauen in Not

\section{DMG Wissenschaftspreise}

- „Neues aus der Wissenschaft" und "Christian Lauritzen Preis"

\section{Kongresspräsidenten/In und} wissenschaftliche Leitung

Prof. Dr. Thomas Römer, Köln

Prof. Dr. Vanadin Seifert-Klauss, München

Prof. Dr. Peyman Hadji, Frankfurt/Main

Deutsche Menopause Gesellschaft e.V.

E-Mail: info-dmg@email.de

www.menopause-gesellschaft.de

\section{Kongressorganisation/Veranstalter}

Softconsult

Weißdornweg 17

D-35041 Marburg/Lahn

Tel.: +49 (0)6420 - 93444

E-Mail: softconsult@web.de

www.soft-consult.org 